\title{
Tutorías académicas y Prácticas Profesionales: invitación al desempeño profesional inicial en la formación trabajadores sociales
}

\author{
Academic tutorials and Professional Practices: invitation to initial \\ professional performance in social workers training
}

\author{
YASNA ANABALÓN ANABALÓN \\ Trabajadora Social \\ Doctoranda en Educación en Consorcio, Becaria/ Doctorado Nacional/ 2017/ Folio \\ CONICYT 21170133 \\ Tutora de prácticas de la Escuela de Trabajo Social \\ Universidad del Bío-Bío, sede Chillán, Chile \\ yanabalon@ubiobio.cl
}

\section{MG. MARCELA CONCHA TORO}

Académica de la Escuela de Trabajo Social

Universidad del Bío-Bío, sede Chillán, Chile

maconcha@ubiobio.cl

\section{MARCELA MORA DONOSO}

Coordinadora Pedagógica de la Unidad de Gestión Curricular

Universidad del Bío-Bío, sede Chillán, Chile

mamora@ubiobio.cl

\section{RESUMEN}

En Chile, las universidades adscritas al Consejo de Rectores abordan diversas iniciativas en función de la innovación curricular, es así como este artículo tiene como objetivo comunicar el proceso metodológico de la tutoría académica de pregrado de la Escuela de Trabajo Social, Universidad del Bío-Bío en el marco de las prácticas profesionales. En el escenario de las prácticas profesionales, las tutorías académicas adquieren relevancia en la formación disciplinaria, entendiendo estas como "una actividad de carácter formativo que incide en el desarrollo integral de los estudiantes universitarios en su dimensión intelectual, académica, profesional y personal" (Ferrer, 2003: 67). Los resultados de aprendizaje que 
permiten alcanzar estos escenarios curriculares son: evidenciar un ejercicio profesional inicial, desde la fundamentación de las metodologías utilizadas en la intervención y su sistematización, siendo procesos fundados, situados y reflexivos, contribuyendo al desarrollo humano y valoración integral de los sujetos de intervención. Es así como se presentan las tutorías académicas como una metodología de acompañamiento en las prácticas profesionales, destacando las fases del proceso de enseñanza-aprendizaje y dispositivos metodológicos utilizados tanto por el docente-tutor como por el estudiante, con énfasis en la reflexión de su propio desempeño.

Palabras clave: formación profesional, tutoría académica, enseñanza-aprendizaje, prácticas profesionales, Trabajo Social.

\begin{abstract}
In Chile, the universities attached to the Council of Rectors address various initiatives based on curricular innovation, so this article aims to communicate the methodological process of undergraduate academic tutoring of the School of Social Work of Bío-Bío University within the framework of professional practices. In the scenario of professional practices, academic tutorials acquire relevance in the disciplinary training, understanding these as "an activity of a formative nature that affects the integral development of university students in their intellectual, academic, professional and personal dimensions" (Ferrer, 2003: 67). The learning outcomes that allow these curricular scenarios to be achieved are: evidencing an initial professional exercise, from the foundation of the methodologies used in the intervention and its systematization, being well-founded, situated and reflexive processes, contributing to the human development and integral valuation of the subjects of intervention. This is how the academic tutorials are presented as a methodology of accompaniment in professional practices, highlighting the phases of the teaching-learning process and methodological devices used by both the teacher-tutor and the student, with emphasis on the reflection of their own performance.
\end{abstract}

Key words: vocational training, academic tutoring, teaching-learning, professional internships, Social Work. 


\section{INTRODUCCIÓN}

La educación universitaria, en especial las universidades adscritas al Consejo de Rectores de las Universidades Chilenas, desarrollaron diversas iniciativas en función de la innovación curricular ( $\mathrm{CRUCH}, 2012)$, asumiendo como desafíos las tendencias internacionales de formación, así como los contextos de desempeño profesional, que por naturaleza son complejos y dinámicos. Asimismo, es imprescindible generar cambios en el currículum, puesto que surgen nuevos enfoques en las disciplinas, producto de los hallazgos en investigaciones, actualizaciones del punto de vista tecnológico y, junto a ello, nuevas prácticas basadas en metodologías activas, lo que implica generar un tránsito del currículum academicista a uno centrado en el aprendizaje, tal como lo establecen los modelos educativos universitarios, concebidos para implementar estos nuevos cambios y reconceptualizar el aprendizaje y la enseñanza.

Por tanto, la necesidad de transformar los itinerarios formativos, tal como lo plantean OCDE (1998), Delors (1996), Rollin (1997), Pey, Chauriye y Chiuminatto (2002), Bernasconi y Rojas (2004), CRUCH (2013), es propio de sistemas que reflexionan sobre su misión y visión formativa, caracterizándose además por poseer capacidad de transformarse (CRUCH, 2012).

Si se compara el currículum antiguo basado en contenidos con el actual currículum basado en competencias y materializado en resultados de aprendizaje, en la estructura de su formulación: verbo, objeto, condición, se evidencia que en esta última se esbozan cuáles son las intenciones pedagógicas del docente y el conjunto de líneas curriculares del plan de estudio en la formación. Collis y Moonen (2011) sostienen que debe ser un proceso de co-construcción con mayor interacción con y entre los estudiantes, a fin de que ambos sujetos participen y sean entes activos del proceso de aprendizaje para desenvolverse de manera adecuada en el quehacer profesional, a partir de la demostración de seguridad, dominio y extrapolación de conocimientos y habilidades sociales desarrolladas en el ámbito académico universitario. En este mismo contexto, González, García y Ramírez (2015) aluden al hecho de que los procesos de enseñanza aprendizaje en el ámbito educativo conllevan al desarrollo e implementación de metodologías activas, repercutiendo en el desarrollo de los estudiantes a nivel personal, familiar y social.

En consecuencia, allí radica la importancia de estas nuevas propuestas curriculares en la formación de competencias genéricas y disciplinarias o 
específicas, que en su conjunto se amalgaman para formar el perfil de egreso de los trabajadores sociales.

\section{DESARROLLO}

\section{Tutorías académicas: desde una mirada de las prácticas profesionales}

La formación académica profesional de los estudiantes de la Escuela de Trabajo Social está caracterizada por el desarrollo de habilidades duras y blandas en permanente reflexión crítica, fundada y reflexiva. Igualmente, el autoaprendizaje cobra relevancia en la capacitación del individuo para la vida profesional, a partir de los ámbitos constitutivos del ser humano. De acuerdo con lo anterior, es que se pone de manifiesto la importancia de desarrollar tutorías académicas en educación superior, en la que se deje de lado la concepción tradicional de la tutoría, centrada solo en resolver dudas de una asignatura específica, a fin de dar cabida a una tutoría más amplia, de carácter orientador, que incluya la vida académica de los estudiantes de la Escuela de Trabajo Social.

Lobato y Guerra (2014, p.18) afirman que la tutoría académica es fundamental en el contexto universitario porque se visualiza como "la intervención educativa personalizada de acompañamiento, asesoramiento y apoyo en la adquisición y maduración de las competencias y en la configuración del proyecto personal y profesional del estudiante".

En este mismo sentido, Cano (2009) sostiene que la tutoría debiera ser el elemento básico de calidad educativa, expresada en sus diversas formas (académica, personal y/o profesional) y en sus diferentes manifestaciones (individual, grupal y entre pares), pues todas ellas son complementarias entre sí. Desde esta perspectiva metodológica, se define la tutoría académica universitaria como una actividad de carácter formativo que incide en el desarrollo integral de los estudiantes universitarios en su dimensión intelectual, académica, profesional y personal (Ferrer, 2003).

Por otro lado, las tutorías académicas a nivel individual y grupal que está desarrollando la Escuela de Trabajo Social de la Universidad del Bío-Bío centran su accionar en el asesoramiento metodológico de los estudiantes que cursan asignaturas anuales de práctica profesional en sus distintos niveles, contribuyendo con ello al desarrollo integral de los alumnos en la praxis profesional que desarrolla 
la Escuela, potenciando y reforzando de manera positiva las fortalezas y, al mismo tiempo, visibilizando y superando las debilidades, desde una mirada horizontal. Por ende, se concuerda con Andreucci y Curiche (2017) que las tutorías académicas se sustentan y se deben realizar bajo el modelo de educación-profesionalización, en el que la comunicación e interacción entre estudiantes y tutores académicos es activa en todo momento durante el proceso de formación profesional.

Además, el estudiante debe: aprender a aplicar en contexto, resolver problemas y conocer ciertas técnicas de actuación profesional que le permitan transferir los aprendizajes adquiridos a su vida académica, profesional y personal, contribuyendo de esta forma a la adaptación profesional en los distintos escenarios y áreas de intervención a desarrollar.

\section{Prácticas profesionales: una invitación al desempeño profesional inicial en la formación de trabajadores sociales}

Las prácticas profesionales del plan de estudio con renovación curricular, que surge el año 2013, son: Práctica Profesional I (correspondiente a tercer año), Práctica Profesional II (en cuarto año) y la Práctica Profesional (de quinto año), situándose esta última en el marco de la asignatura Actividad de Titulación. Estas cátedras son de carácter anual y se adscriben a la línea curricular de intervención social. En ellas se desarrollan acciones de formación, con niveles de logro inicial, intermedio y final, por ende, los estudiantes deben cumplir funciones establecidas por las instituciones/organizaciones en las que se encuentran insertos, desarrollando procesos de intervención social, fundados, situados y reflexivos, contribuyendo así al desarrollo humano y valoración integral de los sujetos de intervención. Las áreas de desempeño en las que se insertan los estudiantes en formación profesional de Trabajo Social son: infancia y adolescencia, justicia, municipio, salud, bienestar, educación, organismos no gubernamentales (ONG), vivienda, género, investigación y de índole territorial/comunitaria.

Esta línea curricular de intervención social, dentro de la cual se incluye las prácticas, tributa a las competencias específicas y subcompetencias del perfil de egreso en la formación de trabajadores sociales, tal como se observa en la siguiente tabla: 


\section{Tabla 1}

Competencias especificas y subcompetencias del área de intervención en contexto de renovación curricular.

\begin{tabular}{ll}
\hline CE3 & $\begin{array}{l}\text { Intervenir en situaciones sociales de distintos niveles de complejidad, } \\
\text { promoviendo el desarrollo humano integral, valorando al ser humano en su } \\
\text { individualidad y en su carácter de sujeto colectivo. }\end{array}$ \\
\hline SC3.1 & $\begin{array}{l}\text { Formular propuestas de intervención situadas y fundamentadas teóricas, } \\
\text { técnica y políticamente. }\end{array}$ \\
\hline SC3.2 & $\begin{array}{l}\text { Aplicar los procedimientos metodológicos del Trabajo Social desde una } \\
\text { perspectiva ética de responsabilidad, compromiso y respeto a la diversidad } \\
\text { sociocultural. }\end{array}$ \\
\hline SC3.3 $\begin{array}{l}\text { Evaluar las intervenciones sociales realizadas para mejorar los procesos y } \\
\text { resultados derivados de la práctica. }\end{array}$ \\
\hline SC3.4 $\begin{array}{l}\text { Actuar profesionalmente de acuerdo a los principios y valores de los derechos } \\
\text { humanos. }\end{array}$ \\
\hline SC3.5 $\begin{array}{l}\text { Desarrollar el trabajo interdisciplinario como parte constitutiva de la intervención } \\
\text { social. }\end{array}$ \\
\hline
\end{tabular}

Fuente: Proyecto Innovación Curricular, Escuela de Trabajo Social (2012).

Asimismo, las siguientes competencias genéricas, contenidas en el Modelo Educativo de la Universidad del Bío-Bío, se asumen en la innovación curricular, constituyendo el marco general que establece la base conceptual global para la docencia, siendo un instrumento clave de gestión curricular y de desarrollo de la política institucional en el proceso de formación. 
Tabla 2

Competencias genéricas.

\begin{tabular}{ll}
\hline № & Competencias Genéricas \\
\hline CG1 & $\begin{array}{l}\text { DISPOSICIÓN PARA EL APRENDIZAJE: Manifestar una actitud permanente } \\
\text { de búsqueda y actualización de sus aprendizajes, incorporando los cambios } \\
\text { sociales, científicos y tecnológicos en el ejercicio y desarrollo de su profesión. }\end{array}$ \\
\hline CG2 & $\begin{array}{l}\text { RESPONSABILIDAD SOCIAL: Asumir un rol activo como ciudadano y } \\
\text { profesional, comprometiéndose de manera responsable con su medio social, } \\
\text { natural y cultural. }\end{array}$ \\
\hline CG3 & $\begin{array}{l}\text { TRABAJO COLABORATIVO: Establecer relaciones dialogantes para el } \\
\text { intercambio de aportes constructivos con otras disciplinas y actúa éticamente } \\
\text { en su profesión. Trabaja de manera asociativa en la consecución de objetivos. }\end{array}$ \\
\hline CG4 & $\begin{array}{l}\text { CAPACIDAD EMPRENDEDORA Y LIDERAZGO: Manifestar convicción para } \\
\text { innovar en su área, toma decisiones y asume riesgos. Ejerce su condición } \\
\text { de liderazgo, potenciando las capacidades de las personas y/o grupos para } \\
\text { alcanzar objetivos deseados. }\end{array}$ \\
\hline CG5 & $\begin{array}{l}\text { CAPACIDAD PARA COMUNICARSE: Comunicar ideas y sentimientos en } \\
\text { forma oral y escrita para interactuar efectivamente en el entorno social y } \\
\text { profesional en su lengua materna y en un nivel inicial en un segundo idioma. }\end{array}$ \\
\hline
\end{tabular}

Fuente: Vicerrectoría Académica. Modelo Educativo de la Universidad del Bío-Bío (2009).

En cuanto a la evaluación, la Escuela de Trabajo Social ha diseñado los siguientes instrumentos de evaluación acordes con cada nivel formativo, a fin de lograr los resultados de aprendizaje propuestos en los programas de las asignaturas de práctica y las competencias específicas del perfil de egreso. 


\section{Tabla 3}

Instrumentos evaluativos según nivel de práctica.

\begin{tabular}{ll}
\hline Nivel de práctica & Instrumentos \\
\hline Práctica Profesional I & $\begin{array}{l}\text { Prácticos; bitácoras de aprendizaje; autoevaluación; } \\
\text { evaluación de desempeño tutor y guía institucional; } \\
\text { pautas de orientación y rúbricas evaluación proyecto de } \\
\text { intervención. }\end{array}$ \\
\hline Práctica Profesional II & $\begin{array}{l}\text { Avances orales de prácticos en aula; bitácoras de } \\
\text { aprendizaje; autoevaluación; evaluación de desempeño } \\
\text { tutor y guía institucional, pautas de orientación y rúbricas } \\
\text { evaluación proyecto de intervención. }\end{array}$ \\
\hline Práctica Profesional & $\begin{array}{l}\text { Pauta de orientación informe ejecutivo } 1 \text { y } 2 \text { (Diagnóstico } \\
\text { (Actividad de Titulación) }\end{array}$ \\
$\begin{array}{l}\text { Situacional, Plan de intervención y Evaluación Plan de } \\
\text { intervención); Evaluación de desempeño } 1 \text { y } 2 \text { (tutor y guía } \\
\text { institucional). }\end{array}$ \\
\hline
\end{tabular}

Fuente: elaboración propia (2017).

\section{Proceso metodológico de la tutoría académica}

Las siguientes fases que se presentan corresponden a la metodología de las tutorías académicas de la Escuela de Trabajo Social, la cual surge a partir de diversas jornadas de reflexión, sostenidas por el equipo de docentes-tutores de prácticas, en las cuales se analizan las fases del proceso, desde un enfoque centrado tanto en el aprendizaje del estudiante como en la enseñanza, relevando el rol del docente-tutor.

\section{Fase 1: Centrada en el Aprendizaje: Reflexión, integración y resignificación de la praxis (permanente y transversal a todas las fases)}

\section{a) Aproximación al proceso de intervención/actuación profesional}

La actividad reflexiva se constituye en una estrategia de aprendizaje, monitoreo y seguimiento cíclico del proceso de práctica. Significa reflexionar permanentemente en torno a la integración teórico-práctica y al proceso metodológico del Trabajo Social, para identificar, sistematizar, deconstruir y evaluar los aprendizajes logrados. 
En esta fase, los estudiantes se sitúan en contexto y área de desempeño profesional; reconocen los marcos conceptuales, epistemológicos y/o teóricos que están presentes en la intervención/actuación profesional, así como los recursos institucionales existentes y a los que se podría acceder; identifican y problematizan las necesidades de los sujetos; reflexionan en torno a su rol como estudiantes en el espacio profesional; reconocen los recursos personales, necesidades y expectativas de aprendizaje en torno al proceso de práctica; y aplican técnicas y metodologías para aproximarse al contexto institucional o territorial.

\section{b) Construcción propuesta de intervención/actuación profesional}

En esta fase, el estudiante reflexiona permanentemente sobre el contexto institucional, área de desempeño profesional, sujetos de atención, propuesta de intervención/actuación y acerca de su rol como agente de intervención. Las principales acciones son: identificación y evaluación de los aprendizajes relevantes en torno a lo anterior (metacognición = qué he aprendido). Asimismo, se espera que el estudiante genere una propuesta creativa que responda a las necesidades identificadas a partir del conocimiento institucional o del espacio territorial, y que problematice las necesidades reconocidas en su aproximación al ámbito de intervención/actuación profesional. Igualmente, se identifica y reflexiona sobre las posibles propuestas de intervención/actuación en torno a las necesidades detectadas (el qué); se define, describe y fundamenta desde la epistemología, teoría, metodología y la práctica, la propuesta elegida (por qué); se identifican los niveles de intervención que se abordarán en la propuesta (individuos, familias, grupos, comunidades y/o territorios); se determinan los objetivos, metas, actividades, metodologías, técnicas, recursos, plazos, tipos y modelos evaluación asociados a la propuesta y a otras intervenciones/actuaciones profesionales (para qué, cómo, con qué); se caracteriza a los sujetos de intervención/actuación de la propuesta (para quién y con quién); y se fundamenta la implementación de la propuesta.

\section{Fase 2: Centrada en la enseñanza: reflexión, integración y resignificación de la praxis (permanente y transversal a todas las fases)}

\section{a) Aproximación al proceso de intervención/actuación profesional}

En esta fase se produce un primer acercamiento con los estudiantes en tutorías de práctica, por ello se realiza un encuadre con respecto a lo que significa insertarse 
en un espacio institucional o territorial; se rescata, reformula y/o refuerzan sus ideas previas y expectativas respecto a las prácticas; también: se reflexiona en torno al concepto de intervención/actuación profesional; se revisan los marcos conceptuales, epistemológicos y/o teóricos que subyacen a la intervención/ actuación profesional; se activan conocimientos previos; se contextualizan los conocimientos en la práctica y se ejercitan las técnicas de actuación profesional. Asimismo, se facilitan en las tutorías de práctica instancias de reflexión en torno a la integración teórico-práctica y al proceso metodológico del Trabajo Social para identificar, sistematizar, deconstruir, evaluar los aprendizajes logrados y desarrollar acciones de mejora pertinentes.

\section{b) Construcción propuesta de intervención/actuación profesional}

Esta fase permite: revisar elementos técnicos para la construcción de la propuesta; facilitar instancias de reflexión; retroalimentar; activar conocimientos previos de tipo metodológicos y teóricos para su construcción; demostrar la relevancia de la conexión entre la propuesta de intervención y los marcos conceptuales, epistemológicos y/o teóricos que están a la base; revisar y retroalimentar los análisis que realiza el estudiante sobre su vinculación con los marcos conceptuales, epistemológicos y/o teóricos.

\section{Enfoque disciplinario en el contexto de las prácticas profesionales}

En los últimos años, se ha producido una renovación de los discursos teóricos y epistemológicos relativos a la intervención social, que es posible evidenciar a partir de la revisión de tres propiedades que emergen de la conceptualización discursiva de la intervención social, a saber: el carácter fundado, situado y reflexivo del fenómeno (Saavedra, 2010).

La noción de intervención social fundada, desarrollada por Matus (2005), implica resignificar el Trabajo Social, al situarse en un horizonte de intervención que tenga como fundamento una rigurosa y compleja comprensión social, y que considere la relación dialéctica entre teoría y praxis.

La intervención social situada responde a las circunstancias, contextos y significados en las que se manifiestan las actuaciones del Trabajo Social frente a demandas de solución de los problemas y conflictos de intereses que 
se evidencian en la sociedad. Saavedra (2010) refiere que, al igual que en el plano de lo fundado, el marco situacional está elaborado desde la modernidad, reconociendo los efectos microsociales en los que se sujeta la acción del Trabajo Social.

De acuerdo con Montenegro (2001), lo situado refiere a aquello digno de transformación. Se define en fijaciones temporales y precarias de significados a partir de las articulaciones de diferentes posiciones de sujeto, por tanto, implica la elaboración un ejercicio de operacionalización de la problemática social, orientado a delimitar el marco espacio-temporal en el cual se hace posible el cambio social propuesto e intencionado.

Por último, el carácter de la intervención social reflexiva relocaliza al Trabajo Social en los territorios de la epistemología socio-crítica de las ciencias sociales, produciendo un desplazamiento desde el positivismo hacia formas que observan el desarrollo del Trabajo Social como articulador del cambio social. Del mismo modo, Castañeda (2014) destaca la importancia de la reflexión y generación de conocimientos desde la práctica, relevando los aprendizajes emergentes desde una praxis asentada en la transformación social.

\section{CONCLUSIONES}

Las tutorías académicas, en el marco de la renovación curricular de la Escuela de Trabajo Social de la Universidad del Bío-Bío, se han constituido en una instancia académica de alta exigencia y nivel formativo en el ámbito de las prácticas profesionales de los estudiantes de la carrera. Esto implica un proceso permanente de acompañamiento y asesoramiento metodológico, al ser un soporte elemental de la calidad educativa que contribuye a una adecuada inserción laboral y al desarrollo integral de los futuros trabajadores sociales en las diversas áreas del desempeño profesional. Ese acompañamiento permite: potenciar y reforzar de manera positiva las fortalezas, visibilizar y superar las debilidades observadas en la formación inicial, mejorar la formación integral de los estudiantes, facilitar la transmisión cultural y contribuir al desarrollo de la educación chilena y la investigación, formando personas capaces de desenvolverse en un escenario cambiante y complejo, que exige el desempeño de múltiples roles, el compromiso constante y la responsabilidad ética en el ejercicio profesional. 
La metodología de las tutorías académicas se desarrolla desde una mirada horizontal, reforzando las habilidades y experiencias de aprendizaje que los estudiantes vivencian en contexto, centrándose, por tanto, en el aprendizaje. Ese proceso está en sintonía con el modelo educativo de la Universidad, puesto que se fortalecen las competencias específicas del trabajador social durante la formación inicial y se incrementan las competencias genéricas.

En relación con el rol del docente tutor, se relevan las acciones de acompañamiento, retroalimentación continua, asesoría, apoyo, orientación, contención emocional, e intencionan evaluaciones formativas y metacognitivas que permiten la reflexión sobre las actuaciones y experiencias profesionales que vivencian los estudiantes en los contextos de prácticas.

Finalmente, surge como desafío la necesidad de complementar el proceso de tutoría con investigaciones que den cuenta de la percepción de los estudiantes, tutores, guías de práctica institucional, académicos de la Escuela y demás actores involucrados, a fin de evaluarlo y perfeccionarlo en función de los posibles nudos críticos o debilidades que surjan para, de esta forma, avanzar hacia su mejoramiento continuo.

\section{REFERENCIAS BIBLIOGRÁFICAS}

ANDREUCCI, P. Y CURICHE, A. (2017). Tutorías académicas: Desafíos de un programa piloto entre pares en una universidad no selectiva. Revista Latinoamericana de Ciencias Sociales, Niñez y Juventud, 15 (1), 357-371.

BERNASCONI, A. Y ROJAS, F. (2004). Informe sobre la educación superior en Chile: 1980- 2003. Santiago, Chile: Editorial Universitaria.

CANO, R. (2009). Tutoría universitaria y aprendizaje por competencias. ¿Cómo lograrlo?. Revista Electrónica Interuniversitaria de Formación del Profesorado, 12(1), 181-204.

CASTAÑEDA, P., (2014). Propuestas metodológicas para Trabajo Social en Intervención Social y Sistematización. Valparaíso: Universidad de Valparaíso, Chile. 
COLLIS, B. Y MOONEN, J. (2011). Flexibilidad en la educación superior: Revisión de expectativas. Comunicar, (37), 15-25.

CONSEJO DE RECTORES DE LAS UNIVERSIDADES CHILENAS [CRUCH]. (2012). Innovación Curricular en las Universidades del Consejo de Rectores. Reflexiones y procesos en las Universidades del Consejo de Rectores. Prácticas Internacionales. Editorial Universidad de Valparaíso.

CONSEJO DE RECTORES DE LAS UNIVERSIDADES CHILENAS, CRUCH. (2013). Manual para la implementación de Sistema de Créditos Académicos Transferibles. SCT-Chile. Santiago de Chile: Consejo de Rectores de las Universidades Chilenas.

DELORS, J. (1996). La educación encierra un tesoro. Informe a la UNESCO de la Comisión internacional sobre la educación para el siglo XXI. Madrid, España: Santillana/UNESCO.

FERRER, V. (2003). La acción tutorial en la Universidad. En: F. Michavila \& J. García (coords.), La Tutoría y los nuevos modelos de aprendizaje en la Universidad. Madrid: Consejería de Educación de la Comunidad Autónoma de Madrid/Cátedra UNESCO de Gestión Política Universitaria de la Universidad Politécnica de Madrid, 67-84.

GONZÁLEZ, N., GARCÍA, R., Y RAMÍREZ, A. (2015). Aprendizaje cooperativo y tutoría entre iguales en entornos virtuales universitarios. Estudios pedagógicos (Valdivia), 41(1), 111-124. https://dx.doi.org/10.4067/S0718.

LOBATO, C. Y GUERRA, N. (2014). Las Tutorías universitarias en el contexto europeo. Orientación y sociedad, 14, 00.

MATUS, T. (2005). Propuestas contemporáneas en Trabajo Social: hacia una intervención polifónica. Buenos Aires: Espacio.

MONTENEGRO, M., (2001). Conocimientos, agentes y articulaciones. Una mirada situada a la intervención social. Athenea Digital, 0. Barcelona: U. Autónoma de Barcelona.

ORGANIZACIÓN DE COOPERACIÓN Y DESARROLLO ECONÓMICOS [OCDE]. (1998). La educación en una mirada: indicadores OCDE 1998. Comisión Económica para América Latina y el Caribe. México. 
PEY, R., CHAURIYE, S. Y CHIUMINATTO, P. (2002). Calidad en la Educación. Consejo Superior de Educación. Diciembre.

ROLLIN, K. (1997). Los temas críticos de la Educación Superior en América Latina en los años 90. Volumen 2. México. ANUIES.

SAAVEDRA, J. (2010). Configuración discursiva en la intervención social fundada, situada y reflexiva. Trabajo Social Cuadernos de Trabajo Social, 6, pp. 49-50. 